\title{
The field theory of Skyrme lattices in quantum Hall ferromagnets
}

\author{
M. Abolfath ${ }^{1,2,3}$, and M. R. Ejtehadi ${ }^{3}$ \\ ${ }^{1}$ Department of Physics, Indiana University, Bloomington, Indiana 47405 \\ ${ }^{2}$ Center for Theoretical Physics and Mathematics, P.O.Box 11365-8486, Tehran, Iran \\ ${ }^{3}$ Institute for Studies in Theoretical Physics and Mathematics P.O. Box 19395-1795, Tehran, \\ Iran
}

(November 22, 2013)

\begin{abstract}
We report the application of the nonlinear $\sigma$ model to study the multiskyrmion problem in the quantum Hall ferromagnet system. We show that the ground state of the system can be described by a ferromagnet triangular Skyrme lattice near $\nu=1$ where skyrmions are extremely dilute. We find a transition into antiferromagnet square lattice by increasing the skyrmion density and therefore $|\nu-1|$. We investigate the possibility that the square Skyrme lattice deforms to a single skyrmion with the same topological charge when the Zeeman energy is extremely smaller than the Coulomb energy. We explicitly show that the energy of a skyrmion with charge two is less than the energy of two skyrmions each with charge one when $g \leq g_{c}$. By taking the quantum fluctuations into account, we also argue the possibility of the existence of a non-zero temperature Kosterlitz-Thouless and a superconductor-insulator phase transition.
\end{abstract}

Typeset using REVTEX 


\section{INTRODUCTION}

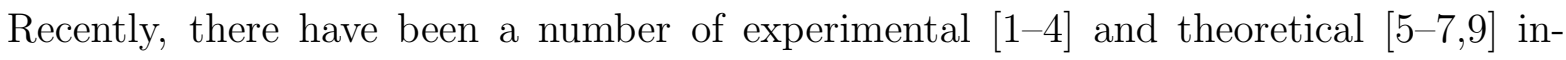
vestigations of the skyrmions in the integer and fractional quantum Hall effect (QHE) in 2D electron gas (2DEG). It has been shown [10,11,24, 27] that the ground state of a twodimensional electron system at the Landau level filling factor near $\nu=1$ is a Skyrme (soliton) lattice. Following Sondhi et al. [5] and Moon et al. [6], one may show that the quantum Hall ferromagnet can be described by an effective nonlinear $\sigma$ (NL $\sigma$ ) model. In this article we also make use of the effective field theory of the quantum Hall NL $\sigma$ model [5, 6, 9, 11] to study a multi-skyrmion quantum Hall system. Our results for a multi-skyrmion system show that the ground state has long range order. We take the advantage of particle-hole symmetry [8] (which is equivalent to skyrmion-antiskyrmion duality in classical field theory) to study the skyrmions associated with holes $(\nu \leq 1)$. We will show that within $\nu_{1 c}<\nu \sim 1$, the ground state is a triangular lattice in order to reduce the Coulomb energy since, the Zeeman energy is negligible. Increasing the skyrmion density in such a way that $0<<\nu_{2 c}<\nu<\nu_{1 c}$, leads to the formation of a square lattice. The square lattice is favored by the gradient and Zeeman energy in order to allow the antiferromagnetic alignment of the skyrmion's orientation via the attractive XY interaction between them. This can be anticipated by the frustration of the skyrmionic hedgehog fields within the triangular lattice which is the favorable orientation for the Coulomb energy. This leads to a structural phase transition due to varying the Landau level filling factor. By taking the quantum fluctuations into account, we also argue the possibility of the existence of a non-zero temperature Kosterlitz-Thouless and a superconductor-insulator phase transition. We show that the 2DES with the typical Zeeman energy at filling factor smaller than one (the validity range of the square lattice) are in the insulating phase. The attractive XY interaction makes a tendency for collapsing

the single skyrmions which are located in different positions. It favors recombination of the $N$-seperated single skyrmions and forming a single skyrmion with topological charge $N$. The competition between attractive XY interaction and repulsive Coulomb interaction 
leads to the stability of the multi-skyrmion system in some circumstances. More explicitly, we investigate this for a special two single skyrmions system numerically. We demonstrate that in a special two single-skyrmion case at extremely small values of the Zeeman energy, (depends on the filling factor) collapse can occur through the formation of a single skyrmion with the topological charge two.

\section{AN OVERVIEW ON NL $\sigma$-MODEL IN QHF}

In the NL $\sigma$-model approach to QHF, the spin of an electron may be described classically by a unit vector whose direction may be changed continuously in the space. In this representation, the effective Hamiltonian is functional of the unit vector $\mathbf{m}(\mathbf{r})$ [5.6]

$$
E[\mathbf{m}]=E_{0}[\mathbf{m}]+E_{z}[\mathbf{m}]+E_{c}[\mathbf{m}]
$$

where $E_{0}[\mathbf{m}]$ and $E_{z}[\mathbf{m}]$ are the conventional NL $\sigma$ model and the Zeeman energy respectively. The last term $E_{c}[\mathbf{m}]$ is the Coulomb energy due to the connection between excess electric charge density and skyrmion topological density [5,6]

$$
\begin{gathered}
E_{0}[\mathbf{m}]=\frac{\rho_{s}}{2} \int d^{2} r(\nabla \mathbf{m})^{2}, \\
E_{z}[\mathbf{m}]=\frac{\tilde{g}}{2 \pi \ell_{0}^{2}} \int d^{2} r\left[1-m_{z}(\mathbf{r})\right], \\
E_{c}[\mathbf{m}]=\frac{e^{2}}{2 \epsilon} \int d^{2} r \int d^{2} r^{\prime} \frac{\rho(\mathbf{r}) \rho\left(\mathbf{r}^{\prime}\right)}{\left|\mathbf{r}-\mathbf{r}^{\prime}\right|} .
\end{gathered}
$$

Here $\rho_{s}=e^{2} /\left(16 \sqrt{2 \pi} \epsilon \ell_{0}\right)$ is the spin stiffness (assuming zero layer thickness for the 2DEG) which arises from the Coulomb exchange energy, $\epsilon$ is the background dielectric constant of the semiconductor, $\tilde{g}=g e^{2} /\left(2 \epsilon \ell_{0}\right)$ is the Zeeman term, $g$ is the effective gyromagnetic ratio, and $\ell_{0}$ is the magnetic length. The charge density is given by [5, 6] $\rho(\mathbf{r})=\frac{-\nu}{8 \pi} \epsilon_{\alpha \beta} \mathbf{m}(\mathbf{r})$. $\left[\partial_{\alpha} \mathbf{m}(\mathbf{r}) \times \partial_{\beta} \mathbf{m}(\mathbf{r})\right]$ which is equal to the filling factor times the topological $O(3)$ spin texture density of the quantum Hall ferromagnet (QHF). The total skyrmion charge denoted by $Q$ 
is an integer-valued topological invariant. It can be determined by the integration upon the charge density and classified by homotopy group of a 2D-sphere respectively.

The lowest energy skyrmion solution, $\tilde{\mathbf{m}}(\mathbf{r})$, has to satisfy a non-linear differential equation which can be obtained by minimizing the energy in Eq.(11) with respect to $\mathbf{m}$

$$
\rho_{s}\left(-\nabla^{2}+\tilde{\mathbf{m}} \cdot \nabla^{2} \tilde{\mathbf{m}}\right) \tilde{\mathrm{m}}_{\mu}-\frac{\tilde{g}}{2 \pi \ell_{0}^{2}}\left(\delta_{z \mu}-\tilde{\mathrm{m}}_{z} \tilde{\mathrm{m}}_{\mu}\right)-\frac{\nu}{4 \pi} \epsilon_{\alpha \beta}\left\{\partial_{\alpha} V(\mathbf{r})\right\}\left(\tilde{\mathbf{m}} \times \partial_{\beta} \tilde{\mathbf{m}}\right)_{\mu}=0,
$$

where $V(\mathbf{r})$ is Hartree potential (the exchange potential resides in $\rho_{s}$ )

$$
V(\mathbf{r})=\frac{e^{2}}{\epsilon_{0}} \int d \mathbf{r}^{\prime} \frac{\tilde{\rho}\left(\mathbf{r}^{\prime}\right)}{\left|\mathbf{r}-\mathbf{r}^{\prime}\right|}
$$

and $\tilde{\rho}$ is the skyrmion charge density associated with the minimum energy solution, $\tilde{\mathbf{m}}(\mathbf{r})$. The solutions of Eq.(3) can be classified by the skyrmion charge $Q=\int d \mathbf{r} \rho(\mathbf{r})$. From now and for the sake of simplicity, we remove the tilde over the classical solution and denote it by $\mathbf{m}$. It is easy to find the following equation of motion of the optimal skyrmion by making use of cross product of $\mathbf{m}$ upon Eq.(3)

$$
\partial_{\alpha} J_{\alpha}^{\lambda}=\frac{\tilde{g}}{2 \pi \ell_{0}^{2}}(\hat{z} \times \mathbf{m})_{\lambda}
$$

where

$$
J_{\alpha}^{\lambda}=\rho_{s}\left(\mathbf{m} \times \partial_{\alpha} \mathbf{m}\right)_{\lambda}-\frac{\nu}{4 \pi} V(\mathbf{r}) \epsilon_{\alpha \beta} \partial_{\beta} m_{\lambda} .
$$

One may immediately read off from Eq.(50) that $\partial_{\alpha} J_{\alpha}^{3}=0$. We may define the ground state of QHF at $\nu=1$ as a vacuum of skyrmionic spin textures where all spins are aligned along the magnetic field direction, i.e. the $\hat{z}$-axis. Note that in the absence of the Zeeman energy, the alignment of spins along an arbitrary axis occurs due to spontaneous global $O(3)$ symmetry breaking [6] hence the minimizing the electrons exchange Coulomb energy. This is also the state of spins far from the center of the skyrmions. Therefore the number of skyrmions (antiskyrmions) is counted by $\left|N-N_{\phi}\right|$.

Before we consider a lattice of skyrmions we need to have the correct shape of a single skyrmion. A single skyrmion is a topological optimal solution of Eq.(团) with $Q=1$. For our purposes, it is convenient to parameterize the unit vector $\mathbf{m}$ by 


$$
\mathbf{m}=\left(\varphi_{x}, \varphi_{y}, \sqrt{1-\bar{\psi} \psi}\right)
$$

where $\psi=\varphi_{x}+i \varphi_{y}$. Near the core of the skyrmion we do not expect that the shape of the skyrmion is influenced much by the magnetic field. However, for large distances from the core, the Zeeman energy becomes dominant and we need to consider its effect where the direction of the unit vector $\mathbf{m}$ is close to the vacuum, namely, $\hat{z}$-axis. Taking the limit of small $\psi$, we can expand the $\mathbf{m}$ up to quadratic order in the $\psi$. Putting this in Eq.(17) gives

$$
E[\psi]=\int d \mathbf{r}\left(\frac{-\rho_{s}}{2} \bar{\psi} \nabla^{2} \psi+\frac{\tilde{g}}{4 \pi \ell_{0}^{2}} \bar{\psi} \psi\right),
$$

which leads to the equation of motion

$$
-\rho_{s} \nabla^{2} \psi+\frac{\tilde{g}}{2 \pi \ell_{0}^{2}} \psi=0
$$

Introducing $\kappa^{2}=\tilde{g} /\left(2 \pi \ell_{0}^{2} \rho_{s}\right)$ we simply have the equation $-\nabla^{2} \psi+\kappa^{2} \psi=0$. We are interested in the 'vortex' solution $\psi=2 \partial_{z} \xi$, where $\partial_{z}=\left(\partial_{x}+i \partial_{y}\right) / 2$ and $z=x+i y$. (If $\tilde{g}=0$, this would result in $\nabla^{2} \xi=0$ with the solution $\xi \propto \ln (r)$ and therefore $\left.\psi \propto z / r^{2}\right)$. Substituting this we find $-\nabla^{2} \xi+\kappa^{2} \xi=0$ or $\xi \propto e^{-\kappa r} / \sqrt{r}$ and therefore that $\psi \propto z e^{-\kappa r} / r^{3 / 2}$ for $r \rightarrow \infty$. The most important of this part is of course the exponential (as opposed to, algebraic if $\tilde{g}=0$ ), fall-off [20]. The dynamics of a skyrmion spin texture in NL $\sigma$-model may be incorporated via the Wess-Zumino action [13]. The result of expansion for the single skyrmion's Wess-Zumino term is

$$
S_{W Z}=\frac{\hbar}{4 \pi \ell_{0}^{2}} \int d \tau \int d t \mathbf{m} \cdot\left(\partial_{\tau} \mathbf{m} \times \partial_{t} \mathbf{m}\right)=\frac{\hbar}{8 \pi \ell_{0}^{2}} \int d t \int d \mathbf{r} \bar{\psi}(\mathbf{r}, t) i \frac{\partial}{\partial t} \psi(\mathbf{r}, t),
$$

where we keep the quadratic terms in Eq.(10). At this level of approximation, the effective action may be obtained by Eq.(8) and Eq.(10) where the Lagrangian density is

$$
\mathcal{L}=\bar{\psi}(\mathbf{r}, t)\left(\frac{\hbar}{8 \pi \ell_{0}^{2}} i \frac{\partial}{\partial t}+\frac{\rho_{s}}{2} \nabla^{2}-\frac{\tilde{g}}{4 \pi \ell_{0}^{2}}\right) \psi(\mathbf{r}, t),
$$

and $S=\int d t \int d \mathbf{r} \mathcal{L}$. The optimal solution of $\psi$ is then identical to the solution of the time dependent Schrödinger equation where the external potential is proportional to the Zeeman splitting factor. Then the single skyrmion behaves approximately like a quantum mechanical point particle far from its core. 


\section{SKYRMION INTERACTION}

We can now consider the interaction between skyrmions by generalizing our linearized energy functional. For that one should note that for the optimal single skyrmion spin texture in Eq. (3) we may choose a particular orientation. In general the texture can be rotated without costing any energy. Since the system shows the $U(1)$ symmetry, any valid skyrmion spin texture can be obtained by rotating all spins about the $\hat{z}$-axis by angle $\chi$. Therefore the state, $\mathbf{m}^{\prime}=\exp \left(i \chi \hat{I}_{z}\right) \mathbf{m}$, is also an optimal solution of the Hamiltonian then $\hat{I}_{z} \equiv-i \partial / \partial \chi$ is the generator of the rotation along the $\hat{z}$-axis in the internal space. One may expect that it contributes to the Hamiltonian through $\left(1 / 2 \Lambda_{0}\right)\left(-i \partial / \partial \chi-\xi_{0}\right)^{2}$ where $\Lambda_{0}$ is the moment of inertia of the single skyrmion. This is the leading term of the total energy which is expanded with respect to the number of reversed spin, $\xi$. In general the dimensional analysis shows $E_{z} \propto \xi$ and $E_{c} \propto 1 / \sqrt{\xi}$. The optimal value of the number of reversed spin, $\xi_{0}=E_{z} /(2 \tilde{g})$, can be evaluated by minimization of the total energy with respect to $\xi$

$$
\xi_{0} \equiv \frac{1}{4 \pi \ell_{0}^{2}} \int d \mathbf{r}\left[1-m_{z}(\mathbf{r})\right]
$$

where $m_{z}(\mathbf{r})$ is the optimal solution of Eq.(3) 9] corresponding to the given Zeeman splitting factor. This leads to the optimal Coulomb and Zeeman energy and then the predicition $E_{c} / E_{z}=2$ hence $\Lambda_{0} \equiv\left(d^{2} E / d \xi^{2}\right)_{\xi_{0}}^{-1}=E_{z} /\left(6 \tilde{g}^{2}\right)$. Our goal is now to calculate the interaction energy between skyrmions with different orientations. Following Piette et al. [17, we start with the conventional NL $\sigma$ model to find out the proper superposition rule for skyrmions. In the absence of the Zeeman and Coulomb energies, the energy functional is scale invariant and one may find the optimal solutions analytically. In this case, it can be shown that any analytic complex polynomial defines an optimal solution [14 16

$$
\mathbf{m}(\mathbf{r})=\left(\frac{2 w_{x}}{1+|w|^{2}}, \frac{2 w_{y}}{1+|w|^{2}}, \frac{1-|w|^{2}}{1+|w|^{2}}\right)
$$

where $w=w_{x}+i w_{y}$ is any $Q$-sector analytical function. One may decompose $w$ into a series of analytical functions each with $Q=1$ 


$$
w=\sum_{j=1}^{N} u_{j} .
$$

Any $Q=1$ analytical function represent a single-skyrmion, then the number of skyrmions $N$ is clearly the total skyrmions topological charge, i.e. $N=Q$. Eq.(14) denotes a sequence of order parameters $\mathbf{m}_{j}(\mathbf{r})$ in configuration space. It is convenient to parameterize the $\mathbf{m}_{j}(\mathbf{r})$ by

$$
\mathbf{m}_{j}(\mathbf{r})=\left(\sin \eta_{j}(\mathbf{r}) \cos \zeta_{j}(\mathbf{r}), \sin \eta_{j}(\mathbf{r}) \sin \zeta_{j}(\mathbf{r}), \cos \eta_{j}(\mathbf{r})\right)
$$

where $\eta_{j}(\mathbf{r})$ and $\zeta_{j}(\mathbf{r})$ are polar and azimuthal field variable associated with $j$ th skyrmion. One may define [9,17] $\zeta_{j}=\varphi-\chi_{j}$ where $\varphi$ is the standard azimuthal angle, e.g. $\mathbf{r}=$ $(r \cos \varphi, r \sin \varphi)$ and $\chi_{j}$ is skyrmions orientation and measuring the deviation from the standard hedgehog fields. If we have a gas of skyrmions far away from each other, the total energy is invariant under variation of skyrmions orientation. For finite separation we expect a coupling between skyrmions due to different orientations. Here we consider a situation where $\mathbf{m}_{i}$ are localized and well separated. This is valid for dilute skyrmions in a quantum Hall system, e.g. $\nu_{2 c}<\nu<\nu_{1 c}$. One may divide the configuration space $\left(\mathrm{R}^{2}\right)$ into $N$ regions such that $u_{i}$ is significant in region $i$ and small in others

$$
u_{i \mu}=\frac{\mathbf{m}_{i \mu}}{1+\hat{z} \cdot \mathbf{m}_{i}}
$$

Here $\mu=(x, y)$ and

$$
\mathbf{m}_{j}=\left(\varphi_{x}^{j}, \varphi_{y}^{j}, \sqrt{1-\Phi_{j} \cdot \Phi_{j}}\right)
$$

where $j \neq i$ and $\Phi_{j}=\left(\varphi_{x}^{j}, \varphi_{y}^{j}\right)$

$$
u_{j \mu}=\frac{\varphi_{\mu}^{j}}{1+\sqrt{1-\Phi_{j} \cdot \Phi_{j}}} .
$$

Substituting Eq.(14)-Eq.(18) into Eq.(13) and expanding $\mathbf{m}_{j}$ up to $\varphi_{j}$, we may find $\mathbf{m}$ in $i$ th region

$$
\mathbf{m}=\mathbf{m}_{i}+\Omega_{i} \times \mathbf{m}_{i}+\frac{1}{2} \Omega_{i} \times\left(\Omega_{i} \times \mathbf{m}_{i}\right)+\mathcal{O}\left(\Omega^{3}\right)
$$


here Eq.(19) describes an infinitesimal rotation of $\mathbf{m}_{i}$ about $\Omega_{i}$ axis where

$$
\Omega_{i}=\frac{1}{2} \mathbf{m}_{i} \times\left\{\left(1+\hat{z} \cdot \mathbf{m}_{i}\right) \Phi_{\mathrm{eff}}^{i}-\left(\mathbf{m}_{i} \cdot \Phi_{\mathrm{eff}}^{i}\right) \hat{z}\right\}
$$

and

$$
\Phi_{\mathrm{eff}}^{i}(\mathbf{r})=\sum_{j \neq i}^{N} \Phi_{j}(\mathbf{r})
$$

Therefore the effect of the other skyrmions on the specific skyrmion is the same as a single skyrmion with charge $Q-1$ via the effective linear field, $\Phi_{\text {eff }}$. In order to study the effect of the Zeeman and Coulomb energy, we make the ansatz that the above superposition rule is valid for skyrmions far from each other even in the presence of full interaction. This can be taken into account by Eq.(19) and evaluating the total energy of multi-skyrmion in QHF. It is obvious that the total energy can be divided into energies in separated regions. We assume that the interaction between the skyrmions is weak, hence $\Omega_{i}=\mathbf{m}_{i} \times \Phi_{\text {eff }}^{i}$. One may obtain easily the total energy by redoing the same calculation for all separated regions and sum over energies

$$
E[\mathbf{m}]=\sum_{i=1}^{N}\left(E\left[\mathbf{m}_{i}\right]+\int_{i} d \mathbf{r} \bar{\psi}_{\mathrm{eff}}^{i}\left\{-\frac{\rho_{s}}{2} \nabla^{2}+\frac{\tilde{g}}{4 \pi \ell_{0}^{2}}\right\} \psi_{\mathrm{eff}}^{i}\right)+V_{\mathrm{eff}}[\mathbf{m}],
$$

where $\psi_{\text {eff }}^{i} \equiv \Phi_{x \text { eff }}^{i}+i \Phi_{y \text { eff }}^{i}$ and $E_{\text {eff }}^{C}[\mathbf{m}]$ is the effective Coulomb interaction between skyrmions. Note that in the absence of the Coulomb term, the saddle point solution associated with the scalar field, $\psi^{i}$, are vortices, i.e. $-\nabla^{2} \psi^{i}+\kappa^{2} \psi^{i}=0$. One may divide the total energy, Eq.(22), into two parts, the self energy of skyrmions and interaction energy which are designated by $T$ and $V$ respectively

$$
T[\mathbf{m}]=\sum_{i=1}^{N}\left(E\left[\mathbf{m}_{i}\right]+\int_{i} d \mathbf{r} \sum_{j \neq i}^{N} \bar{\psi}^{j}\left\{-\frac{\rho_{s}}{2} \nabla^{2}+\frac{\tilde{g}}{4 \pi \ell_{0}^{2}}\right\} \psi^{j}\right) .
$$

The first term in Eq.(23) is the total self energy of the isolated skyrmions and the second term is the effect of their tail in other regions, i.e., the contribution of their kinetic energy in regions far from the core. Here we are interested to study the effect of interaction in a system of many skyrmions and their physical relevance. One may find the effective interaction 
between the skyrmions by making use of the Stokes theorem and neglecting the next nearest neighbor terms in Eq.(22) which are described by the terms like $\int_{i} d \mathbf{r} \sum_{j \neq i} \sum_{k \neq j} \Phi_{j} \cdot \Phi_{k}$ and $\int_{i} d \mathbf{r} \sum_{j \neq i} \sum_{k \neq j} \partial_{\alpha} \Phi_{j} \cdot \partial_{\alpha} \Phi_{k}$ then

$$
V_{\mathrm{eff}}[\mathbf{m}]=E_{\mathrm{eff}}^{0}[\mathbf{m}]+E_{\mathrm{eff}}^{C}[\mathbf{m}]
$$

and

$$
E_{\mathrm{eff}}^{0}[\mathbf{m}]=\sum_{i=1}^{N} \int_{i} d \mathbf{r} \partial_{a}\left\{J_{a}^{\lambda(i)} \Omega_{i \lambda}\right\}=\frac{\rho_{s}}{2} \sum_{<i j>} \int_{i} d \mathbf{r} \bar{\psi}^{j}\left\{\nabla^{2}-\kappa^{2}\right\} \psi^{i},
$$

where $\mathbf{J}^{(i)}$ has been defined for $i$ th skyrmion by Eq.(6). $E_{\text {eff }}^{0}[\mathbf{m}]$ is the contribution of the gradient and Zeeman energy to the effective interaction. It describes a system of interacting dipoles. Expanding the charge density of skyrmions, $\rho$, in terms of $\psi$, leads to the effective Coulomb interaction

$$
\begin{gathered}
E_{\mathrm{eff}}^{C}[\mathbf{m}]=\frac{e^{2}}{2 \epsilon_{0}} \sum_{i \neq j} \int_{i} d \mathbf{r} \int_{j} d \mathbf{r}^{\prime} \frac{\rho_{i}(\mathbf{r}) \rho_{j}\left(\mathbf{r}^{\prime}\right)}{\left|\mathbf{r}-\mathbf{r}^{\prime}\right|} \\
+\epsilon_{\mu \nu} \frac{\nu e^{2}}{4 \pi \epsilon_{0}} \sum_{i \neq j}\left\{\int_{i} d \mathbf{r} \partial_{\mu} V^{j}(\mathbf{r}) \partial_{\nu} \mathbf{m}_{i} \cdot \Omega_{j}+\int_{j} d \mathbf{r} \partial_{\mu} V^{i}(\mathbf{r}) \partial_{\nu} \mathbf{m}_{j} \cdot \Omega_{i}\right\},
\end{gathered}
$$

where $\rho_{i}=\frac{-\nu}{8 \pi} \epsilon_{\alpha \beta} \mathbf{m}_{i} \cdot\left[\partial_{\alpha} \mathbf{m}_{i} \times \partial_{\beta} \mathbf{m}_{i}\right]$. The first and second term in Eq.(26) are the Coulomb energy due to the monopole and dipole counterparts of skyrmions respectively. The former falls off like $R^{-1}$ whereas the latter falls as $R^{-2}$ where the distance between two skyrmions is denoted by $R$. Unlike the dense skyrmions in which the effect of the dipole term is crucial, the monopole term dominates for an extremely dilute (i.e. $\nu \simeq 1$ ) skyrmions system. Neglecting the Coulombic dipole term, one may evaluate the integrals in Eq.(25) by applying the techniques that were developed for a pair of skyrmions by Piette et al. [17

$$
V_{\mathrm{eff}}[\mathbf{m}]=\frac{e^{2}}{2 \epsilon_{0}} \sum_{i \neq j} \int_{i} d \mathbf{r} \int_{j} d \mathbf{r}^{\prime} \frac{\rho_{i}(\mathbf{r}) \rho_{j}\left(\mathbf{r}^{\prime}\right)}{\left|\mathbf{r}-\mathbf{r}^{\prime}\right|}+\frac{c^{2} \tilde{g}}{4 \pi^{2}} \sum_{<i j>} \cos \left(\chi_{j}-\chi_{i}\right) K_{0}\left(\kappa\left|\mathbf{R}_{j}-\mathbf{R}_{i}\right|\right),
$$

where $\chi_{j}-\chi_{i}$ and $R_{j}-R_{i}$ describe the in-plane relative orientation and effective distance between skyrmion $j$ th and $i$ th. The first term in Eq.(27) is the electrostatic monopole term independent of the relative orientation. The normalization factor, $c=2 \sqrt{2}$, is calculated for a single skyrmion. The second term in Eq.(27) describes a classical XY-model where the 
minimum energy configuration specifies the relative orientation corresponds to $\chi_{j}-\chi_{i}=\pi$. $K_{0}(x)$ is the modified Bessel function, hence the coupling between the site $i$ and $j$ decays exponentially. We see that there is an exponential decrease of the coupling between the $i$ th and $j$ th skyrmions for $R \gg 1 / \kappa$. The XY term of Eq.(27) favors forming a Q-skyrmion $(Q=N)$ by recombining the $N$ single skyrmions, hence the collapse of the lattice. (Note that the sign of this term in the antiferromagnet ordering is negative, therefore smaller seperation between skyrmions, $R$, is favorable.) Such a combination costs the Coulomb energy. The global minimum solution of the energy functional can be identified by the skyrmions self energy and the interaction terms. Apart from the self energy terms which leads to an important effect to the recombination of the single skyrmions, one has to take the interaction terms into account to predict the proper critical point of this transition. Since the Coulomb interaction is long range and the XY term contributes to the total energy as a short range interaction, then an antiferromagnet ordering within a square lattice can be the global minimum of the total energy, and no combining takes place except for small values of the Zeeman energy in agreement with the recent result of Lilliehöök et al. [25]. We examine this effect by considering a special case, e.g. two skyrmions system. However, finding the global minimume of the total energy for the general $N$-skyrmion system as a function of the filling factor and the Zeeman splitting factor needs more investigation. An estimate based on Eq.(27) shows that the energy of a skyrmion with charge two is lower than the energy of two skyrmions each with charge one. Note that the inter skyrmionic XY and Coulomb energies are canceled out if $g \sim|\nu-1|^{1 / 3}$ at the limit of $\nu \rightarrow 1$. Let's consider a system of two single skyrmions with charge one. The total energy of this system can be obtained by employing Eq.(27) into Eq.(22). One may compare the total energy of the two single skyrmions with the self energy of a skyrmion with charge two in order to estimate the critical value of the Zeeman energy

$$
E[\mathbf{m} ; Q=2]=2 E[\mathbf{m} ; Q=1]+\frac{e^{2}}{2 \epsilon_{0}} \int_{1} d \mathbf{r} \int_{2} d \mathbf{r}^{\prime} \frac{\rho_{1}(\mathbf{r}) \rho_{2}\left(\mathbf{r}^{\prime}\right)}{\left|\mathbf{r}-\mathbf{r}^{\prime}\right|}-\frac{c^{2} \tilde{g}}{4 \pi^{2}} K_{0}\left(\kappa\left|\mathbf{R}_{1}-\mathbf{R}_{2}\right|\right),
$$


where $E[\mathbf{m} ; Q]$ is the self energy of the classical Q-skyrmion. This can be evaluated by Eq.(11) [25]. Results of our numerical calculation for $\tilde{g}_{c}$ for several $\nu$ are listed in Table $\llbracket$. The values of the spin stiffness corresponding to the different fractional filling factors, have been evaluated by Moon et al. [6] numerically using the hypernetic chain approximation. We expect that our expression for the inter skyrmionic XY interaction, Eq.(28), fails for filling factors, $|\nu-1|>4 \pi\left(\kappa \ell_{0}\right)^{2}$ due to increasing the density of the skyrmions $(\kappa R \sim 1)$. At $\nu=1$, the inter skyrmionic interaction is exactly zero and one may find $\tilde{g}_{c}=0.53 \times 10^{-5}$ in agreement with the Lilliehöök et al. [25]. At $\nu=1$, the competition between self energies yields the transition, since the interaction terms do not play role in this case. However, at $\nu<1$ the value of $\tilde{g}_{c}$ is enhanced by the skyrmion interaction. Since the order of the Coulomb energy and the short range XY interaction with respect to the number of particles are $\mathcal{O}\left(N^{2}\right)$ and $\mathcal{O}(N)$ respectively, then the effective energy of a multi-skyrmion system, Eq.(27), becomes dominant by Coulomb energy, hence the stability of the lattice at the limit of large $\mathrm{N}$ as a local minimum is guaranteed. For larger separations, the $\mathrm{XY}$ term of Eq.(27) is therefore negligible compared to the effective Coulomb energy. This implies that for a lower density of skyrmions the Coulomb interaction determines the lattice structure and leading to a triangular lattice. However, for a more dense system one may expect a different stable structural symmetry, in agreement with the calculation of Brey et al. [10]. One may define a domain of separation for which the square lattice is valid, e.g. $\nu_{2 c}<\nu<\nu_{1 c}$ or $\kappa^{-1}<R<R_{0}$. Here the length scale cut off is denoted by $R_{0}$, beyond that a phase transition to a triangular lattice occurs. One may consider the static and dynamical properties of a skyrmion lattice [20] using the interaction that we have derived

$$
V\left(\left|\mathbf{R}_{i}-\mathbf{R}_{j}\right| ; \chi_{i}-\chi_{j}\right)=V_{0}\left(\left|\mathbf{R}_{i}-\mathbf{R}_{j}\right|\right)+\cos \left(\chi_{i}-\chi_{j}\right) V_{1}\left(\left|\mathbf{R}_{i}-\mathbf{R}_{j}\right|\right) .
$$

In our model $V_{0}(R)$ is the electrostatic monopole interaction and $V_{1}(R)=\frac{c^{2} \tilde{g}}{4 \pi^{2}} K_{0}(\kappa R)$. This is consistent with the result of variational calculation [20,24,28]. Obviously, the skyrmion density may be controlled via the Landau level filling factor. As mentioned above, unlike the case where the competition between monopole Coulomb term and the gradient dipole 
terms is crucial to the stability of the square lattice, the electrostatic monopole interaction is the dominant term to determine the structure of the lattice for an extremely dilute $(\nu \sim 1)$ skyrmions. In this situation a specific skyrmion moves in a background which is being made by the vacuum of the other skyrmions. Since $u_{i}$ which has been defined by Eq.(16), is zero every where but in the $i$ th region, then $\rho(\mathbf{r})=\sum_{i} \rho_{i}(\mathbf{r})$. Clearly the total charge is the summation upon the individual skyrmion's charge, i.e. $Q_{\text {tot }}=\sum_{i} Q_{i}$ where $Q_{i}$ is the topological charge of localized skyrmion in that region. In this case, the interaction between separated skyrmions is independent of the relative orientation. The skyrmions may be considered by point particles where $\rho_{i}(\mathbf{r})=\nu \delta\left(\mathbf{r}-\mathbf{R}_{i}\right)$ and

$$
V_{\mathrm{eff}}=\frac{e^{2}}{2 \epsilon_{0}} \sum_{i \neq j}^{N} \int d \mathbf{r} \int d \mathbf{r}^{\prime} \frac{\rho_{i}(\mathbf{r}) \rho_{j}\left(\mathbf{r}^{\prime}\right)}{\left|\mathbf{r}-\mathbf{r}^{\prime}\right|}=\frac{\nu^{2} e^{2}}{2 \epsilon_{0}} \sum_{i \neq j}^{N} \frac{1}{\left|\mathbf{R}_{i}-\mathbf{R}_{j}\right|}
$$

Therefore the ground state is being described by Eq.(30) is clearly a triangular lattice, independent of the skyrmion orientation, i.e., a Wigner crystal [21]. This is in agreement with the result of Green et al. [11.

One may find the critical filling factor $\nu_{1 c}$ where a transition into square lattice takes place. Note that we have made an approximation associated with $R>\kappa^{-1}$ to demonstrate the existence of the square lattice. We may expect the approximation, namely the superposition rule of Eq.(19), fails for a high density skyrmions. The crossover between low and high density of skyrmions occurs at $\kappa R \sim 1$ and leading to the lower critical filling factor $\nu_{2 c}$, i.e. $\nu_{2 c}=1-\tilde{g} / 2 \rho_{s}$. Using the typical Zeeman energy, $\tilde{g}=0.015 e^{2} / \epsilon_{0} \ell_{0}$ for GaAs and taking the advantage of skyrmion-antiskyrmion duality, yields $\left|\nu_{2 c}-1\right| \sim 0.3$. Our estimate for lower limit of $\nu_{c}$ shows good agreement with the result of random phase approximation of Brey et al. [10].

The appropriate quantum mechanical spectrum of the Skyrme lattice in our model can be obtained through Eq.(27) and the Wess-Zumino action

$$
\begin{aligned}
& \left\{\sum_{i=1}^{N}\left[-4 \pi \rho_{s} \ell_{0}^{2}\left(\nabla_{\mathbf{R}_{\mathbf{i}}}^{2}+\kappa^{2}\right)+\frac{1}{2} \Lambda_{0}^{-1}\left(-i \frac{\partial}{\partial \chi_{i}}-\xi_{0}\right)^{2}\right]+\sum_{i \neq j} V_{0}\left(\left|\mathbf{R}_{i}-\mathbf{R}_{j}\right|\right)\right. \\
& \left.+\sum_{<i j>} V_{1}\left(\left|\mathbf{R}_{i}-\mathbf{R}_{j}\right|\right) \cos \left(\chi_{i}-\chi_{j}\right)\right\} \Psi([\mathbf{R} ; \chi] ; t)=i \hbar \frac{\partial}{\partial t} \Psi([\mathbf{R} ; \chi] ; t),
\end{aligned}
$$


where $[\mathbf{R} ; \chi] \equiv\left(\mathbf{R}_{1} ; \chi_{1}, \mathbf{R}_{2} ; \chi_{2}, \ldots, \mathbf{R}_{N} ; \chi_{N}\right)$ and $\mathbf{R}_{i} \equiv\left(R_{i} \cos \varphi_{i}, R_{i} \sin \varphi_{i}\right) . \varphi_{i}$ is the standard azimuthal angle, indicating the $i$ th skyrmion. $\Lambda_{0}=\xi_{0} /(3 \tilde{g})$ is the moment of inertia of a single skyrmion, and $\xi_{0}=E_{z} /(2 \tilde{g})$. Obviously, Eq. 31 describes a quantum rotor problem for $\chi$-degree of freedom [19], consistent with the result of Ref. [27,28]. This model has been used to describe the superconductor-insulator transition in granular superconductors and Josephson junction arrays [18]. We may expect an insulator-superconductor phase transition occurs due to variation of the Zeeman splitting $(\tilde{g})$ at a given filling factor $(\nu<1)$. Using the empirical data for practical systems which are consistent with the experiments of Bayot et al. [4] $\left(\tilde{g}=0.015 e^{2} / \epsilon \ell_{0} \sim 2 K\right.$ at $\left.\nu=0.8\right)$ in which the XY coupling constant, $J=\left(2 \tilde{g} / \pi^{2}\right) K_{0}\left(\sqrt{\tilde{g} / 2 \pi \ell_{0}^{2} \rho_{s}} R\right) \sim 0.05 K$, is a fraction of the $\left(2 \Lambda_{0}\right)^{-1} \sim 0.4 K$ leads to the prediction of the Mott insulating phase for this system where the position of skyrmions are fixed. We also expect that the system go through superconducting phase for $J$ greater than $\Lambda_{0}^{-1}$ since the skyrmions carring the electrical charges. The ground state described by Eq.(31) for skyrmions orientation is a quantum antiferromagnet where the U(1) symmetry is broken spontaneously. Here $E_{z}=0.214 e^{2} / \epsilon \ell_{0}$ is the Zeeman energy associated with the optimal single skyrmion solution corresponding to $\tilde{g}=0.015 e^{2} / \epsilon \ell_{0}$, is evaluated by the solving the non-linear, non-local integro-differential equation Eq.(3), for a single skyrmion numerically. The details of this calculation will be presented elsewhere [9].

The Hamiltonian, Eq.(31), may also lead to a finite temperature Kosterlitz-Thouless phase transition which might be observable. We may predict a critical temperatures for the Kosterlitz-Thouless phase transition based on the classical XY-model. Our calculation for the classical 2D nearest neighbor XY-model on the square lattice, shows that the phase transition occurs around $T_{c}=0.9 \mathrm{~J} \sim 0.04 K$ at $\nu=0.8\left(B_{0} \sim 7 T\right)$ where we consider the square lattice constant, $a=2 \ell_{0} \sqrt{\pi /|\nu-1|}$, and the nearest neighbour separation between skyrmions, $R=a \sqrt{2} / 2$. Recent observations of an anomaly in the heat capacity may support such a phase transition [4,26]. However, this interpretation can be controversial since one may argue that this effect is a remnant of the nuclear Schottky anomaly [19,27]. 


\section{CONCLUSION}

We showed that for extremely dilute skyrmions, the ground state is a triangular lattice to minimize the Coulomb repulsion. However at higher densities, a square lattice forms to optimize the spin gradient and Zeeman energies. The ferromagnet triangular lattice become frustrated by increasing the skyrmion density at critical filling factor $\nu_{c}$ and a transition occurs into an antiferromagnet square lattice. We also argued the possibility of the Kosterlitz-Thouless and superconducting-insulator phase transition. We have shown that in some special cases the multi-skyrmionic system becomes unstable with respect to the formation of the single-skyrmion system with the same conserved topological charge.

\section{ACKNOWLEDGEMENT}

The authors thanks H.A. Fertig, S.M. Girvin, A.H. MacDonald, J.J. Palacios, S. Rouhani and H.T.C. Stoof for helpful discussion. Our special thanks go to S.M. Girvin and H.T.C. Stoof for inspiring comments and ideas on the subject. The work at Indiana University is supported by NSF DMR-9416906. MA would like to thank Indiana University for the warm hospitality. 


\section{REFERENCES}

[1] S. E. Barrett, G.Dabbagh, L.N. Pfeiffer, K.W. West, and R. Tycko, Phys. Rev. Lett. 74, 5112 (1995); R. Tycko, S.E. Barrett, G. Dabbagh, L.N. Pfeiffer, and K.W. West, Science 268, 1460 (1995).

[2] A. Schmeller, J. P. Eisenstein, L. N. Pfeiffer, and K. W. West, Phys. Rev. Lett. 75, $4290(1995)$.

[3] E. H. Aifer, B. B. Goldberg, D. A. Broido, Phys. Rev. Lett. 76, 680 (1996).

[4] V. Bayot, E. Grivei, S. Melinte, M.B. Santos, M. Shayegan, Phys. Rev. Lett. 76, 5484 (1996).

[5] S.L. Sondhi, A. Karlhede, S.A. Kivelson, and E.H. Rezayi, Phys. Rev. B 47, 16419 (1993).

[6] K. Moon, H. Mori, Kun Yang, S.M. Girvin, A.H. MacDonald, L. Zheng, D. Yoshioka, and Shou-Cheng Zhang, Phys. Rev. B 51, 5138 (1995). S. M. Girvin and A. H. MacDonald, in Novel Quantum Liquids in Semiconductor Structures, edited by S. DasSarma and A. Pinczuk (wiley, NewYork, 1996).

[7] Kun Yang, K. Moon, L. Zheng, A. H. MacDonald, S. M. Girvin, D. Yoshioka, and Shou-Cheng Zhang, Phys. Rev. Lett. 72, 732 (1994).

[8] H. A. Fertig, L. Brey, R. Cote, and A. H. MacDonald, Phys. Rev. B 50, 11018 (1994).

[9] M. Abolfath, J. J. Palacios, H. A. Fertig, S. M. Girvin, and A. H. MacDonald, submitted to Phys. Rev. B.

[10] L. Brey, H. A. Fertig, R. Côte, and A. H. MacDonald, Phys. Rev. Lett. 75, 2562 (1995).

[11] A.G. Green, I.I. Kogan, and A.M. Tsvelik, Phys. Rev. B 54, 16838 (1996).

[12] A. M. Polyakov, Phys. Lett. 59B, 79 (1975). J. P. Rodriguez, Phys. Rev. B 39, 2906 (1989). 
[13] Eduardo Fradkin, Field Theories of Condensed Matter Systems (Addison Wesley, 1991).

[14] R. Rajaraman, Solitons and Instantons, (North Holland, Amsterdam, 1982).

[15] A. A. Blavin and A. M. Polyakov, JETP Lett. 22, 245 (1975).

[16] G. Woo, J. Math. Phys. 18, 1264 (1977).

[17] B.M.A.G. Piette, B.J. Schroers, and W.J. Zakrzewski, Z. Phys. C 65, 165 (1995). B.M.A.G. Piette, B.J. Schroers, and W.J. Zakrzewski, Nucl. Phys. B 439, 205 (1995).

[18] Min-Chul Cha, Matthew P.A. Fisher, S.M. Girvin, Mats Wallin, and A. Peter Young, Phys. Rev. B 44, 6883 (1991).

[19] S. M. Girvin, private communication.

[20] H.T.C. Stoof, private communication.

[21] Lynn Bonsall and A. A. Maradudin, Phys. Rev. B 15, 1959 (1977).

[22] Chetan Nayak and Frank Wilczek, Phys. Rev. Lett. 77, 4418 (1996).

[23] Kun Yang and S. L. Sondhi, Phys. Rev. B 54, R2331 (1996).

[24] Madan Rao, Surajit Sengupta, and R. Shankar, preprint cond-mat/9702193.

[25] D. Lilliehöök, K. Lejnell, A. Karlhede, and S.L. Sondhi, preprint, cond-mat/9704121

[26] A.H. MacDonald, preprint, cond-mat/9609283.

[27] R. Côte, A.H. MacDonald, Luis Brey, H.A. Fertig, S.M. Girvin, and H.T.C. Stoof, preprint, cond-mat/9702207.

[28] Yu. V. Nazarov and A.V. Khaetskii, preprint, cond-mat/9703159. 


\section{TABLES}

TABLE I. The critical $\tilde{g}$-factor for several Landau level filling factors are presented. The two single-skyrmions system become unstable at $\tilde{g} \leq \tilde{g}_{c}$ and a transition to a single skyrmion system with charge two occurs.

\begin{tabular}{lcc}
\hline \hline$\nu$ & $\rho_{s}\left(e^{2} / \epsilon \ell_{0}\right)$ & $\tilde{g}_{c}\left(e^{2} / \epsilon \ell_{0}\right)$ \\
\hline 1.0 & $2.49 \times 10^{-2}$ & $0.53 \times 10^{-5}$ \\
$1 / 3$ & $9.23 \times 10^{-4}$ & $0.19 \times 10^{-3}$ \\
$1 / 5$ & $2.34 \times 10^{-4}$ & $0.52 \times 10^{-4}$ \\
\hline \hline
\end{tabular}

